\title{
Extracellular Tau and Glymphatic Influx in Sleep and Anesthesia
}

\author{
J. Shashi Kiran Reddy ${ }^{1}$
}

\begin{abstract}
Affiliations:
${ }^{1}$ Consciousness Studies Programme, National Institute of Advanced Studies (NIAS), IISc Campus, Bengaluru, India.

*Correspondence to: Email Address: jumpalreddy@live.com,jumpal_shashi@yahoo.com
\end{abstract}

\begin{abstract}
Holth and colleagues recently examined how the sleep-wake cycle influence/regulate the amount of metabolic waste, mainly extracellular tau, in the brain (1). They report that interstitial fluid (ISF) tau in mice increases approximately by $90 \%$ during normal wakefulness versus sleep, and $100 \%$ during sleep deprivation (SD) as compared to 50\% increase in CSF tau in humans. During SD, humans also registered increased levels of certain proteins like tau and synuclein with no significant changes in the levels of other neural proteins, suggesting some specificity in sleep/protein level interaction. This indicates that the above changes may be due to increased release of certain proteins rather than changes in global ISF clearance, which authors attribute to elevated neuronal metabolism/synaptic strength during SD and wakefulness (2). A major portion of the extracellular tau in the brain results from high neuronal/synaptic activity $(2,3)$, but, we suggest, other potential factors may also add up to elevate the levels of tau normally present in ISF.
\end{abstract}

\section{One Sentence Summary}

Extracellular tau in sleep and anesthesia 


\section{Main Text:}

Holth and colleagues recently examined how the sleep-wake cycle influence/regulate the amount of metabolic waste, mainly extracellular tau, in the brain (1). They report that interstitial fluid (ISF) tau in mice increases approximately by $90 \%$ during normal wakefulness versus sleep, and $100 \%$ during sleep deprivation (SD) as compared to 50\% increase in CSF tau in humans. During SD, humans also registered increased levels of certain proteins like tau and synuclein with no significant changes in the levels of other neural proteins, suggesting some specificity in sleep/protein level interaction. This indicates that the above changes may be due to increased release of certain proteins rather than changes in global ISF clearance, which authors attribute to elevated neuronal metabolism/synaptic strength during SD and wakefulness (2).

A major portion of the extracellular tau in the brain results from high neuronal/synaptic activity $(2,3)$, but, we suggest, other potential factors may also add up to elevate the levels of tau normally present in ISF. A recent study (4) suggests that different anesthetics, as a common mechanism of action, alter the oscillatory modes in proteins negatively affecting the intra-neuronal microtubule polymerization and function (which propels the production of pathological tau). This cautions one against generalizing the findings during anesthesia to sleep, although some anesthetics may emulate sleep like conditions. While glymphatic influx may serve as a unitary clearance mechanism in the brain parenchyma during both sleep and anesthesia $(5,6)$, various sourcing mechanisms for extracellular toxic waste differs. Thus, as indicated in (4), one should also study the expression of neuronal microtubule during sleep and SD to know how it promotes the release and seeding/spreading of tau. These observations will have explicit relevance to post-operative cognitive dysfunction (POCD) in cytoskeleton-compromised neurodegenerative disorders such as Alzheimer's and Parkinson's. 


\section{References:}

1. J. K. Holth et al., Science 363, 880 (2019).

2. J.W. Wu et al., Nature Neuroscience 19, 1085-1092 (2016).

3. K. Yamada et al., JEM 211,387-393 (2014).

4. T.J.A. Craddock et al., Scientific Reports 7, 9877 (2017).

5. L. Xie et al., Science 342, 373-377 (2013).

6. L. M. Hablitz et al., Sci Adv 5(2) (2019). 